\title{
The effect of food deprivation on escape from electrical stimulation of the VMH
}

\author{
R. IAN HORRELL and PETER REDGRAVE \\ Hull University, Hull, HU $67 R X$, England
}

\begin{abstract}
Rats with permanently implanted intracranial electrodes were trained in a shuttlebox to escape from intermittent electrical stimulation of the ventromedial hypothalamus. Their latency to escape was measured under different degrees of food deprivation $(0,21$, and $45 \mathrm{~h})$. Latency was found to decline with increasing deprivation. It is argued that this finding is evidence against the hypothesis that the structure acts as a "satiety center" in the control food intake.
\end{abstract}

It has long been known that intracranial stimulation (ICS) at sites almost anywhere in the hypothalamus or limbic system may have motivating and reinforcing properties in situations where the occurrence of ICS is contingent upon an animal's behavior (Olds \& Olds, 1963). Typically, this is demonstrated by "self-stimulation" in a Skinner box. It seems reasonable to suppose that these properties are dependent on the normal function of the neural systems activated by the ICS. The function that has been most commonly assigned to the ventromedial nucleus of the hypothalamus (VMH) has been that of a "satiety center" for the control of food intake, collating information on the quality and quantity of food ingested and suppressing further intake when deficits have been made good (Anand \& Brobeck, 1951; Stellar, 1954). Although much evidence has accumulated recently that seems incompatible with this hypothesis and although it is clear that the VMH also has other functions, this hypothesis still has support: several recent reviews of the neural mechanisms subserving the control of food intake continue to attribute to the VMH a specific inhibitory function based on the detection of food intake as the most probable explanation of the effects of lesions or stimulation of the structure on feeding behavior (Baile \& Forbes, 1974; Balagura, 1972; Deutsch \& Deutsch, 1973; Hoebel, 1971; Mogenson \& Huang, 1973). Among the evidence generally quoted in support of this hypothesis is the finding that application of ICS to $\mathrm{VMH}$ results in the cessation of eating, even in food-deprived animals (Anand \& Dua, 1955; Wyrwicka \& Dobrzecka, 1960). Here, excitation by ICS is presumed to achieve a neural state that approximates that normally achieved by the ingestion of

The authors wish to thank the University of Hull for facilities and for personal support for the second author, and Pamela Greathead for technical assistance. The work has been included in a thesis submitted to the University of Hull by the second author for the degree of Master of Science. food. Food intake itself is usually positively reinforcing, especially in a hungry animal, and it might therefore be expected that ICS which acinieves a similar neural state would also be rewarding. A relationship of this kind has indeed recently been found for stimulation of the amygdala (White, 1973).

The actual reinforcing properties of VMH stimulation are somewhat unclear. Early reports (Olds, 1960; Krasne, 1962) that it had a basically aversive character have recently been challenged (Atrens \& von Vietinghoff-Riesch, 1972; Ball, 1972). In favor of a positively reinforcing component, Ball obtained self-stimulation from VMH sites, and Atrens found that, in a box in which ICS was turned on by movement to one end and off at the other, rats would oscillate between the two ends. It may be noted, however, that Ball's rats required unusually long training and that, in the situation used by Atrens, rats chose to spend most of their time with ICS off, in contrast to those with lateral hypothalamic electrodes. In support of the existence of an aversive component, we have found it generally very easy to train rats with VMH electrodes to escape from intermittently applied short bursts of ICS in a shuttlebox. At the present time, it seems appropriate to conclude from these apparently conflicting reports that stimulation of VMH has ambivalent properties, with both aversive and reinforcing components.

The fact that stimulation of the VMH has a substantial aversive component can, however, be readily accommodated by the "satiety center" hypothesis by assuming that either (i) excitation of other processes with which VMH is known to be associated (Paxinos \& Bindra, 1972) is aversive or (ii) VMH is being excited to abnormally high levels, producing an effect equivalent to gross overeating (this is plausible in view of the high frequencies of the usual stimulating waveforms compared to the typically observed firing rates of hypothalamic nerve cells). However, although the existence of aversive effects can be explained, they should be reduced in degree by food deprivation: 
a given excitation (simulating a given food intake) is more likely to be rewarding (and more likely to overcome the aversive effects due to excitation of other systems) and less likely to have the effect of a nauseous excess if the animal is hungry. The experiment described below was designed to investigate the relationship between food deprivation and escape from ICS applied to VMH.

\section{METHOD}

Eight male hooded rats, weighing $200-250 \mathrm{~g}$ at surgery, were implanted unilaterally with monopolar electrodes aimed at VMH under sodium pentobarbital anesthesia $(40 \mathrm{mg} / \mathrm{kg}$ IP). Electrodes were made from 00 ga stainless steel insect pins, insulated except for $0.25 \mathrm{~mm}$ at the tip. A skull screw was used as reference electrode.

Intermittent ICS was supplied by a pulse generator which was set to given monophasic, negative-going, square-wave pulses of duration $0.2 \mathrm{msec}$ and frequency $40 \mathrm{~Hz}$. Pulse trains of $250 \mathrm{msec}$ duration were repeated at a rate of 1 train/sec. Current was continuously monitored by one channel of a two-beam oscilloscope connected across a series resistor; the other channel monitored the potential difference across the rat. Behavioral testing was carried out in a white plastic shuttlebox (area $28 \times 39 \mathrm{~cm}$, with $33 \mathrm{~cm}$ on high sides and a $2.5-\mathrm{cm}$-high barrier across the middle) with a 3-W bulb on each end-wall.
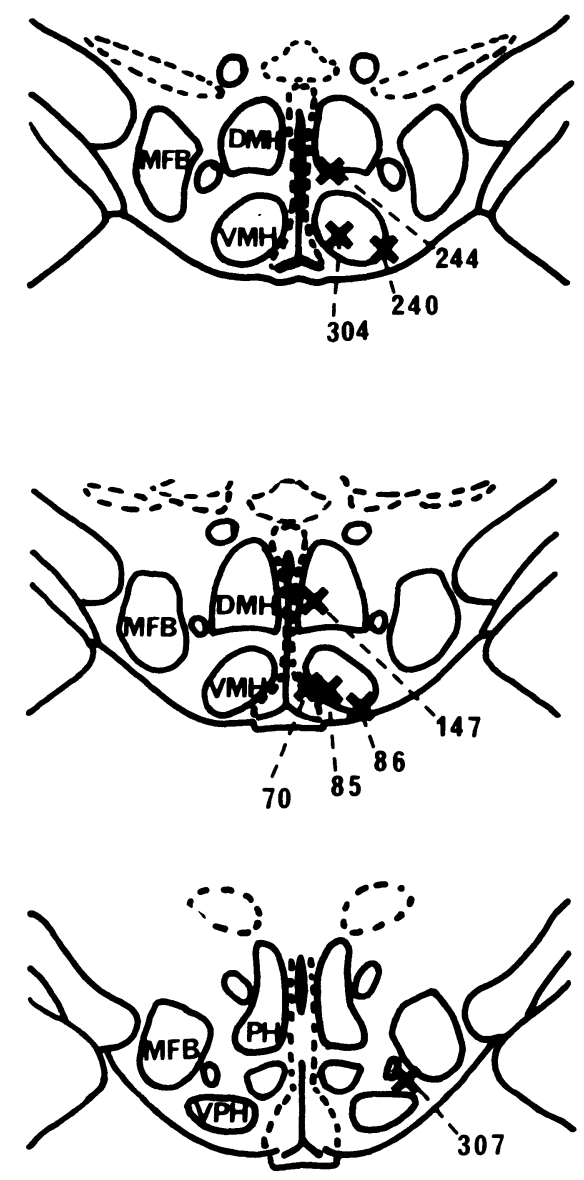

Figure 1. Sites of electrode tips, with rat identification numbers. Structures represented include the dorsomedial hypothalamic nucleus (DMH), the medial forebrain bundle (MFB), the posterior hypothalamic nucleus (PH), the ventromedial hypothalamic nucleus (VMH), and the ventral premamillary nucleus (VPH).

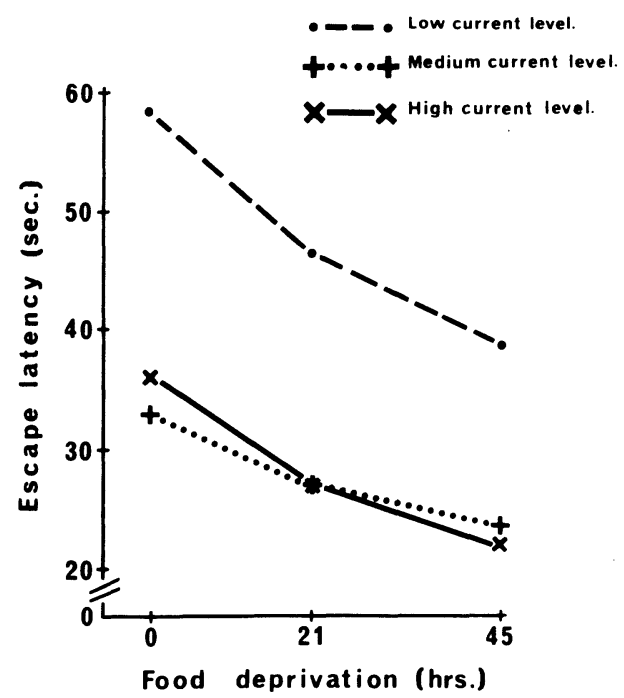

Figure 2. Mean escape latencies for all rats as a function of food deprivation, for each of the three levels of current used.

Four to 7 days after recovery from the operation, the rats were connected to the stimulator, placed in the shuttlebox and trained to escape from ICS by crossing the barrier across its middle. A trial was initiated by switching on the ICS and the lamp on the end-wall farthest from the rat. ICS was terminated when the rat crossed the barrier or, if it failed to escape, after $180 \mathrm{sec}$. Time between onset of ICS and traverse of the barrier ("escape latency") was recorded. A 1-min rest period then supervened before the next trial. Each animal was practised on this task daily for $1 \mathrm{~h}$ until escape latencies stabilized, using different ICS currents to establish an effective range. Rats were also adapted to 21 and $45 \mathrm{~h}$ of food deprivation during this period.

For the experiment proper, three current levels were selected for each rat. For some, a zero current condition was also included in which the trial was as normal except that no current was delivered. Three levels of food deprivation were used: 0,21 , and $45 \mathrm{~h}$. The animals were run on 18 days, giving 6 days of trials at each deprivation level. On each experimental day, the rats were given six trials at each current level. Orders of deprivation condition over days and of current level within days were systematically varied.

After completion of the main experiment, the effect of ICS on feeding was checked in six rats. For this, the food intake of the 23-h deprived animal over a $1 / 2-h$ test period with ICS on continuously was compared with that in a similar period without ICS. For 5 successive days, rats were deprived of food for $23 \mathrm{~h}$ and allowed to eat for $1 \mathrm{~h}$. Food eaten was determined every $15 \mathrm{~min}$ during the feeding period. On Day 3, ICS was applied for the second and third 15-min period and, on Day 4, for the first and fourth. The results presented were derived from data obtained on these 2 days only. Finally, rats were sacrificed and perfused and their brains retained for subsequent histological examination. Electrode placements were determined by taking $50 \mu$ sections on a freezing microtome and staining with potassium ferrocyanide.

\section{RESULTS AND DISCUSSION}

Sites of electrode tips are represented in Figure 1, the mean escape latencies at each food deprivation and current level in Figure 2, and results for individual rats, including the effects of ICS on feeding, in Tables 1 and 2, while latencies in the control conditions in which no current was applied are shown 
Table 1

Escape Latencies for Individual Rats

\begin{tabular}{rcccc}
\hline & & & \multicolumn{3}{c}{$\begin{array}{c}\text { Escape Latencies } \\
\text { (Seconds) }\end{array}$} \\
\cline { 3 - 5 } Rat & Currents Used & & $\begin{array}{c}\text { Food Deprivation } \\
\text { (Hours) }\end{array}$ \\
\hline 70 & (Microamperes) & 0 & 21 & 45 \\
85 & $250,400,500$ & 24 & 23 & 20 \\
86 & $300,400,500$ & 62 & 54 & 41 \\
147 & $500,600,700$ & 97 & 57 & 41 \\
240 & $200,450,700$ & 22 & 20 & 19 \\
244 & $150,325,500$ & 34 & 26 & 20 \\
304 & $400,700,1,000$ & 16 & 17 & 19 \\
307 & $50,350,650$ & 45 & 40 & 42 \\
\hline
\end{tabular}

Note-The columns give, in order, rat identification numbers, the three current levels used (in microamps), and the escape latencies at each of the three levels of food deprivation (latencies for the three current levels pooled).

Table 2

The Effectiveness of Stimulation in Suppressing Feeding in Each Rat

\begin{tabular}{cccccc}
\hline & \multicolumn{2}{c}{$\begin{array}{c}\text { Current } \\
\text { Parameters }\end{array}$} & & \multicolumn{2}{c}{$\begin{array}{c}\text { Food Intake* } \\
\text { (Grams) }\end{array}$} \\
\cline { 2 - 5 } & \multicolumn{3}{c}{$\begin{array}{c}\text { Micro- } \\
\text { Rat }\end{array}$} & & Current \\
\cline { 3 - 6 } Reres & & On & Off \\
\hline 70 & 40 & 250 & & .95 & 2.99 \\
147 & 20 & 200 & & .86 & 3.65 \\
240 & 40 & 325 & & .55 & 3.46 \\
244 & 40 & 400 & & 1.28 & 3.07 \\
304 & 40 & 100 & & 1.02 & 3.13 \\
307 & 20 & 300 & & .09 & 2.34 \\
\hline
\end{tabular}

* for each $1 / 4-h$ test period

Note-The columns give, in order, rat identification numbers, stimulation parameters used (frequency and current), and mean food intake.

Table 3

Escape Latency for Test Periods in Which No Current Was Applied

\begin{tabular}{cc}
\hline $\begin{array}{c}\text { Food Deprivation } \\
\text { (Hours) }\end{array}$ & $\begin{array}{c}\text { Escape Latency } \\
\text { (Seconds) }\end{array}$ \\
\hline 0 & $127( \pm 15.4)$ \\
21 & $104( \pm 15.9)$ \\
45 & $121( \pm 43.6)$ \\
\hline
\end{tabular}

Note-The means for the four animals tested on this condition are followed, in parenthesis, by the standard errors of these means.

in Table 3. Figure 2 shows that, at all current levels used, escape latency decreases with food deprivation, indicating an apparent increase in the aversiveness of VMH stimulation. A three-way analysis of variance was carried out according to the model suggested by Winer (1962) using the mean escape latencies for each rat on each condition as raw data. This showed current $(p<.01)$, food deprivation $(p<.05)$, and differences between individual rats $(p<.001)$ as well as the interactions between individuals and depriva- tion $(\mathrm{p}<.001)$ and between individuals and current $(p<.001)$ to be significant. In view of the significant interaction between food deprivation and individual differences, Table 1 is included to show the results for individual rats: it can be seen that the trend for shorter latencies with food deprivation is evident to some degree in most individuals. The only rat with a contrary trend (No. 244) also showed the least suppression of food intake by ICS despite needing the highest current to show one at all. It may also be noted that rat 307 had a substantially misplaced electrode but still showed clear-cut suppression of feeding by ICS.

It can be seen, then, that the general trend of the results is in the reverse direction to that predicted on the basis of the "satiety center" hypothesis: rats find VMH stimulation increasingly aversive with food deprivation. These results may be regarded as analogous or complementary to the "lateral hypothalamic paradox": stimulation of lateral hypothalamus seems to induce hunger but is rewarding and, moreover, the degree of this rewarding effect, as indicated by self-stimulation rates, increases with hunger (Hoebel, 1969; Margules \& Olds, 1962). Here, activation of a system which is supposed to record food intake not only has a basically aversive component to it, rather than a positively reinforcing one, but seems to be more rather than less aversive when the animal is hungry. The data appear, therefore, to add to the accumulating evidence that is incompatible with the simple "satiety center" hypothesis [see Mogenson, (1974), Panksepp (1974) and Rabin (1972), for reviews and Davis, Nakajima, and White (1974) and Sclafani, Berner, and Maul (1975) for more recent evidence].

Other proposals that have been made to explain the effects of lesions or stimulation of the $\mathrm{VMH}$ on feeding fall into two classes. One class denies it a specific role in the control of food intake but subsumes these efforts under some more general function, like altered affective responsiveness to all kinds of stimuli (Grossman, 1966, 1972) or general response suppression (Margules \& Stein, 1969; Morgane, 1969; Singh, 1973). These would not seem to predict any systematic relationship between food deprivation and the motivating effect of ICS. The other class assigns to the $\mathrm{VMH}$ some modified but, nonetheless, specific function in the control of food intake. Panksepp $(1971,1974)$ has suggested that it operates as a meter of long-term nutrient reserves. But, insofar as activation of the VMH appears to be aversive, if it is initially less active, as it would be in a state of nutrient depletion induced by food deprivation, external activation by ICS superimposed on this should again be less, and certainly not more, aversive. Alternatively, Powley and Keesey (1970), arguing from evidence provided by Hoebel and Teitelbaum (1966), proposed that the VMH forms 
part of a system concerned with fixing the "set point" for body weight and that lesions of the VMH raise the set point level while stimulation lowers it. A corollary of this hypothesis is that any deviation of body weight from the set point should create a drive state from which an animal should work to escape. Food deprivation itself reduces weight, and hence creates a drive to increase it again, while stimulation of the VMH would lower the set point below the present weight level and create a drive to reduce weight. But if, as in this experiment, weight level has already been reduced by food deprivation, ICS should be less drive-inducing or aversive. Thus, both alternative suggestions for a specific function for the VMH in the control of food intake would appear to predict the same trend as does the satiety center hypothesis.

It might be argued that the trend to shorter escape latencies simply reflects the well-documented greater activity of food-deprived animals (Baumeister, Hawkins, \& Cromwell, 1964). However, data on activity in this experiment (escape latency on a trial in which no ICS was applied) gave no evidence of more rapid escape in this condition when food deprived (Table 3). Moreover, in a preliminary experiment in which the effect of a long-term restriction of food intake and consequent weight loss on escape behavior was investigated (Redgrave, 1974), it was found that only a partial component of the systematic variation in latency with weight loss could be attributed to general activity (as indicated by the number of shuttlings during the intertrial rest period). Even if the obtained trend, or at least part of it, is due to a general behavioral activation of this kind, the results do not seem compatible with the simple "satiety center" hypothesis, though they may be with some of the other hypotheses discussed above. On the other hand, looking at the data on individual differences, there seems to be a correlation between the ease of obtaining suppression of eating and the degree of the effect of food deprivation on escape. Moreover, it has been shown that the threshold current for the motivational effects of VMH stimulation in different rats correlates with that for the suppression of food intake (Ball, 1972). If these can be taken to indicate that the changes in aversiveness with food deprivation are directly related to a specific function of $\mathrm{VMH}$ in the control of food intake, no existing hypothesis of this function would seem capable of explaining the results we have obtained.

\section{REFERENCES}

ANAND, B. K., \& BrobeCK, J. R. Hypothalamic control of food intake in rats and cats. Yale Journal of Biology and Medicine, 1951, 24, 123-140.

ANAND, B. K., \& Dua, S. Feeding responses induced by electrical stimulation of the hypothalamus of the cat. Indian Journal of Medical Research, 1955, 43, 113-122.
Atrens, D. M., \& von Vietinghoff-Riesch, F. The motivational properties of the medial and paraventricular hypothalamic nuclei. Physiology and Behavior, 1972, 9, 229-235.

BAILE, C. A., \& Forbes, J. M. Control of food intake and regulation of energy balance in ruminants. Physiological Review's, 1974, 54, 160-214.

Balagura, S. Neurophysiologic aspects: Hypothalamic factors in the control of eating behavior. Advances in Psychosomatic Medicine, 1972, 7, 25-48.

BaLl, G. G. Self-stimulation in the ventromedial hypothalamus. Science, 1972, 178, 72-73.

Baumeister, A., Hawkins, W. F.. \& Cromwell, R. L. Need states and activity level. Psychological Bulletin, 1964, 61, 438-453.

Davies, R., Nakajima, S., \& White, N. Enhancement of feeding produced by stimulation of the ventromedial hypothalamus. Journal of Comparative and Physiological Psychology. 1974, 86, 414-419.

Deutsch, J. A., \& Deutsch, D. Physiological Psychology. Homewood: Dorsey Press, 1973.

Grossman, S. P. The VMH: A center for affective reactions, satiety or both? Physiology and Behavior, 1966, 1, 1-10.

Grossman, S. P. Aggression, avoidance, and reaction to novel environments in female rats with ventromedial hypothalamic lesions. Journal of Comparative and Physiological Psychology. 1972, 78, 274-283.

HoEbel. B. G. Feeding and self-stimulation. Annals of the New York Academy of Science, 1969, 157, 758-778.

Hoebel, B. G. Feeding: Neural control of intake. Annual Review of Physiology, 1971, 33, 533-568.

Hoebel, B. G., \& Teitelbaum, P. Weight control in normal and hypothalamic hyperphagic rats. Journal of Comparative and Physiological Psychology, 1966, 61, 189-193.

KRASNE, F. B. General disruption resulting from electrical stimulation of the ventromedial hypothalamus. Science, 1962 , 138, 822-823.

Margules. D. L., \& Olds, J. Identical feeding and rewarding systems in the lateral hypothalamus of rats. Science, 1962, 135, 374-475.

Margules. D. L.. \& Stein. L. Cholinergic synapses in the ventromedial hypothalamus for the suppression of operant behavior by punishment and satiety. Journal of Comparative and Physiological Psychology, 1969, 67, 327-335.

Mogenson. G. J. Changing views of the role of the hypothalamus in the control of ingestive behaviors. In K. Lederis \& K. E. Cooper, Recent studies of hypothalamic function. Basel: S. Karger. 1974. Pp. 268-293.

Mogenson, G. J., \& Huang, Y. H. The neurobiology of motivated behavior. Progress in Neurobiology, 1973. 1. 53-83.

Morgane. P. J. The function of the limbic and rhinic forebrain - limbic midbrain systems and reticular formation in the regulation of food and water intake. Annals of the New York Academy of Science, 1969, 157, 806-848.

OLDs. J. Approach-avoidance dissociations in rat brain. American Journal of Physiology, 1960, 199. 965-968.

Olds, M. E., \& Olds, J. Approach-avoidance analysis of rat diencephalon. Journal of Comparative Neurology. 1963. 120. 259-295.

Panksepp. J. A re-examination of the role of the ventromedial hypothalamus in feeding behavior. Physiology \& Behavior. 1971, 7, 385-394.

PANKSEPP. J. Hypothalamic regulation of energy balance and feeding behavior. Federation Proceedings, 1974, 33, 1150-1165.

Paxinos, G., \& Bindra, D. Hypothalamic knife cuts: Effects on eating, drinking, irritability, aggression, and copulation in the male rat. Journal of Comparative and Physiological Psychology, 1972, 79, 219-229.

Powley, T. L., \& Keesey, R. E. Relationship of body weight to the lateral hypothalamic feeding syndrome. Journal of Comparative and Physiological Psychology, 1970, 70, 25-36. 
RABIN. B. M. Ventromedial hypothalamic control of food intake and satiety: a reappraisal. Brain Research, 1972 , 43. 317-342.

REDGRAVE, P. The ventromediui hypothalamus: An analysis of ingestive behavior and n'gative reinforcement. Thesis submitted for the degree of Master of Science, University of Hull, 1974.

Sclafani, A., Berner, C. N., \& Maul, G. Multiple knifecuts between the medial and lateral hypothalamus in the rat: A reevaluation of hypothalamic feeding circuitry. Journal of Comparative and Physiological Psychology, 1975, 88, 210-217.

SingH, D. Comparison of behavioral deficits caused by lesions in septal and ventromedial hypothalamic areas of female rats. Journal of Comparative and Physiological Psychology, 1973, 84, 370-379.
Stellar, E. The physiology of motivation. Psychological Review, 1954, 61, 5-22.

White, N. M. Self-stimulaltion and suppression of feeding observed at the same site in the amygdala. Physiology \& Behavior, 1973, 10, 215-219.

WINER, B. J. Statistical principles in experimental design. New York: McGraw-Hill, 1962. Pp. 319-335.

WYRWICKA, W., \& DobrzeCKA, C. Relationship between feeding and satiation centers of the hypothalamus. Science, $1960,132,805-806$.

(Received for publication July 1975 ; accepted February 1976.) 